Krzysztof Ciesielski, Department of Mathematics, West Virginia University, Morgantown, WV 26506-6310, USA. e-mail: KCies@wvnvms. wvnet. edu Saharon Shelah ${ }^{\dagger}$ Institute of Mathematics, the Hebrew University of Jerusalem, 91904 Jerusalem, Israel, and Department of Mathematics, Rutgers University, New Brunswick, NJ 08854, USA

\title{
UNIFORMLY ANTISYMMETRIC FUNCTIONS WITH BOUNDED RANGE
}

\begin{abstract}
The goal of this note is to construct a uniformly antisymmetric function $f: \mathbb{R} \rightarrow \mathbb{R}$ with a bounded countable range. This answers Problem 1(b) of Ciesielski and Larson [6]. (See also the list of problems in Thomson [9] and Problem 2(b) from Ciesielski's survey [5].) A problem of existence of uniformly antisymmetric function $f: \mathbb{R} \rightarrow \mathbb{R}$ with finite range remains open.
\end{abstract}

A function $f: \mathbb{R} \rightarrow \mathbb{R}$ is said to be uniformly antisymmetric [6] (or nowhere weakly symmetrically continuous [9]) provided for every $x \in \mathbb{R}$ the limit $\lim _{n \rightarrow \infty}\left(f\left(x+s_{n}\right)-f\left(x-s_{n}\right)\right)$ equals 0 for no sequence $\left\{s_{n}\right\}_{n<\omega}$ converging to 0. Uniformly antisymmetric functions have been studied by Kostyrko [7], Ciesielski and Larson [6], Komjáth and Shelah [8], and Ciesielski [1, 2]. (A connection of some of these results to the paradoxical decompositions of the Euclidean space $\mathbb{R}^{n}$ is described in Ciesielski [3].) In particular in [6] the authors constructed a uniformly antisymmetric function $f: \mathbb{R} \rightarrow \mathbb{N}$ and noticed that the existence of a uniformly antisymmetric function cannot be proved without an essential use of the axiom of choice.

The terminology and notation used in this note is standard and follows [4]. In particular for a set $X$ we will write $|X|$ for its cardinality and $\mathcal{P}(X)$ for its

Key Words: uniformly antisymmetric function, Hamel basis

Mathematical Reviews subject classification: 26A15, 26A21

Received by the editors May 3, 1998

* The first author was partially supported by NSF Cooperative Research Grant INT9600548, with its Polish part being financed by Polish Academy of Science PAN, and 1996/97 West Virginia University Senate Research Grant.

Papers authored or co-authored by a Contributing Editor are managed by a Managing Editor or one of the other Contributing Editors.

The authors thank the referee for many valuable comments and suggestions.

$\dagger$ This work was supported in part by a grant from "Basic Research Foundation" of the Israel Academy of Sciences and Humanities. Publication 680. 
power set. Also $2^{\omega}$ will stand for the set of all functions from $\omega=\{0,1,2, \ldots\}$ into $2=\{0,1\}$. We consider $2^{\omega}$ as ordered lexicographically.

Theorem 1. There exists a function $f: \mathbb{R} \rightarrow \mathbb{R}$ with countable bounded range such that for every $x \in \mathbb{R}$ there exists an $\varepsilon_{x}>0$ with the property that the set

$$
S_{x}=\left\{s \in \mathbb{R}:|f(x-s)-f(x+s)|<\varepsilon_{x}\right\}
$$

is finite. In particular $f$ is uniformly antisymmetric.

Proof. First notice that it is enough to find a compact zero-dimensional metric space $\langle T, d\rangle$ and a function $g$ from $\mathbb{R}$ into a countable subset $T_{0}$ of $T$ such that for every $x \in \mathbb{R}$ there is a $\delta_{x}>0$ for which the set

$$
\hat{S}_{x}=\left\{s \in \mathbb{R}: d(g(x-s), g(x+s))<\delta_{x}\right\}
$$

is finite.

To see this assume that such a function $g: \mathbb{R} \rightarrow T$ exists and take a homeomorphic embedding $h$ of $T$ into $\mathbb{R}$. We claim that $f=h \circ g: \mathbb{R} \rightarrow \mathbb{R}$ is as desired. Indeed, $f[\mathbb{R}]=h[g[\mathbb{R}]]$ is countable, as it is a subset of a countable set $h\left[T_{0}\right]$, and it is bounded, since it is a subset of a compact set $h[T]$. So take $x \in \mathbb{R}$ and $\delta_{x}>0$ for which $\hat{S}_{x}$ is finite. Since $h^{-1}: h[T] \rightarrow T$ is uniformly continuous, we can find an $\varepsilon_{x}>0$ such that

$$
\left|y_{1}-y_{2}\right|<\varepsilon_{x} \text { implies } d\left(h^{-1}\left(y_{1}\right), h^{-1}\left(y_{1}\right)\right)<\delta_{x}
$$

for every $y_{1}, y_{2} \in h[T]$. But for such a choice of $\varepsilon_{x}$ we have

$$
S_{x}=\left\{s \in \mathbb{R}:|h(g(x-s))-h(g(x+s))|<\varepsilon_{x}\right\} \subset \hat{S}_{x}
$$

proving that $S_{x}$ is finite.

Thus, we proceed to construct a function $g$ described above. The value of $g(x)$ will be defined with help of a representation of $x$ in a Hamel basis; i.e., a linear basis of $\mathbb{R}$ over $\mathbb{Q}$. For this we will use the following notation. Let $\left\{y_{\eta}: \eta \in 2^{\omega}\right\}$ be a one-to-one enumeration of a Hamel basis $\mathcal{H}$. For every $x \in \mathbb{R}$ let $\sum_{\eta \in 2^{\omega}} q_{x, \eta} y_{\eta}$, with $q_{x, \eta} \in \mathbb{Q}$ for $\eta \in 2^{\omega}$, be the unique representation of $x$ in basis $\mathcal{H}$ and let $w_{x}=\left\{\eta \in 2^{\omega}: q_{x, \eta} \neq 0\right\}$. Thus $w_{x}$ is finite and

$$
x=\sum_{\eta \in w_{x}} q_{x, \eta} y_{\eta} .
$$

The definition of the space $T$ is considerably more technical since it reflects several different cases of the proof that the sets $\hat{S}_{x}$ are indeed finite. To this 
end let $\left\{q_{j}: j<\omega\right\}$ be a one-to-one enumeration of $\mathbb{Q}$ with $q_{0}=0$. For $i<\omega$ let $\mathcal{P}_{i}=\mathcal{P}\left(\left\{q_{j}: j<i\right\}\right)$ and put $P_{i}=\mathcal{P}\left(2^{i} \times\{0,1\} \times \mathcal{P}_{i} \times \mathcal{P}_{i}\right)$. Note that each $P_{i}$ is finite; so $T=\prod_{i<\omega} P_{i}$, considered as the standard product of discrete spaces, is compact zerodimensional. We equip $T$ with a distance function $d$ defined between different $s, t \in T$ by $d(s, t)=2^{-\min \{i<\omega: s(i) \neq t(i)\}}$ and let

$$
T_{0}=\{t \in T:(\exists n<\omega)(\forall i \geq n) t(i)=\emptyset\} .
$$

Clearly $T_{0}$ is countable.

Now we are ready to define $g: \mathbb{R} \rightarrow T_{0} \subset T$. For this, however, we will need few more definitions. For $x \in \mathbb{R}, q \in \mathbb{Q}, i<\omega$, and $\zeta \in 2^{i}$ such that $\zeta \in\left\{\left(\eta\lceil i): \eta \in w_{x}\right\}\right.$ we define:

- $p(i) \in\{0,1\}$ as the parity of $i$; i.e., $p(i)=i \bmod 2$;

- $k_{i}(q)=\left\{q_{j} \in \mathbb{Q}: q_{j}<q \& j<i\right\} \in \mathcal{P}_{i}$;

- $\eta(x, \zeta)$ to be the minimum of $\left\{\eta \in w_{x}: \zeta \subset \eta\right\}$ (in the lexicographical order);

- $\xi(x, \zeta)$ to be the minimum of $\left\{\eta \in w_{x}: \zeta \subset \eta\right\} \backslash\{\eta(x, \zeta)\}$ provided $\left|\left\{\eta \in w_{x}: \zeta \subset \eta\right\}\right| \neq 1$; otherwise we put $\xi(x, \zeta)=\eta(x, \zeta)$;

- $n_{x}<\omega$ to be the smallest number $n>0$ such that

(i) $\eta\left\lceil n \neq \xi\left\lceil n\right.\right.$ for any different $\eta, \xi \in w_{x}$, and

(ii) $q_{x, \eta} \in\left\{q_{j}: j<n\right\}$ for every $\eta \in w_{x}$.

Consider the function $g: \mathbb{R} \rightarrow T_{0}$ defined as follows. For every $x \in \mathbb{R}$ and $i<\omega$ we define $g(x)(i) \in P_{i}$ as

$$
\left\{\left\langle\zeta, p\left(\left|\left\{\eta \in w_{x}: \zeta \subset \eta\right\}\right|\right), k_{i}\left(q_{x, \eta(x, \zeta)}\right), k_{i}\left(q_{x, \xi(x, \zeta)}\right)\right\rangle: \zeta \in\left\{\left(\eta\lceil i): \eta \in w_{x}\right\}\right\}\right.
$$

provided $i \leq n_{x}$ and we put $g(x)(i)=\emptyset$ for $n_{x}<i<\omega$. In the argument below the key role will be played by the function $k_{i}$ in general, and the coordinate $k_{i}\left(q_{x, \eta(x, \zeta)}\right)$ in particular.

The key step in the proof that $g$ has the desired property is that for every $x \in \mathbb{R}$ and $s \neq 0$

$$
\text { if } n_{x} \leq \max \left\{n_{x-s}, n_{x+s}\right\} \quad \text { then } g(x-s)\left(n_{x}\right) \neq g(x+s)\left(n_{x}\right) .
$$

To see (1) assume that $n_{x} \leq n_{x+s}$. If $n_{x-s}<n_{x}$, then $g(x-s)\left(n_{x}\right)=\emptyset \neq$ $g(x+s)\left(n_{x}\right)$, where $g(x+s)\left(n_{x}\right) \neq \emptyset$ since $w_{x+s} \neq \emptyset$ as $n_{x-s}<n_{x} \leq n_{x+s}$ implies $x+s \neq 0$. Thus, we can assume that $n_{x} \leq \min \left\{n_{x-s}, n_{x+s}\right\}$. Take an 
$\hat{\eta} \in w_{x-s} \cup w_{x+s}$ such that $q_{x-s, \hat{\eta}} \neq q_{x+s, \hat{\eta}}$ and let $\zeta=\hat{\eta}\left\lceil n_{x}\right.$. Note that, by the definition of $n_{x}$, the set $S=\left\{\eta \in w_{x}: \zeta \subset \eta\right\}$ has at most one element.

If $S=\emptyset$, then $\left\{\eta \in w_{x-s}: \zeta \subset \eta\right\}=\left\{\eta \in w_{x+s}: \zeta \subset \eta\right\} \neq \emptyset$ and so $\eta(x-s, \zeta)=\eta(x+s, \zeta) \notin w_{x}$ while $q_{x-s, \eta(x-s, \zeta)}+q_{x+s, \eta(x+s, \zeta)}=0$. Thus $q_{0}=$ 0 separates $q_{x-s, \eta(x-s, \zeta)}$ and $q_{x+s, \eta(x+s, \zeta)}$ implying that $k_{n_{x}}\left(q_{x-s, \eta(x-s, \zeta)}\right) \neq$ $k_{n_{x}}\left(q_{x+s, \eta(x+s, \zeta)}\right)$. Therefore $g(x-s)\left(n_{x}\right) \neq g(x+s)\left(n_{x}\right)$.

So, assume that $S \neq \emptyset$ and let $\eta^{\prime}$ be the only element of $S$. Then $\eta^{\prime} \in$ $w_{x-s} \cup w_{x+s}$. If $\eta^{\prime}$ belongs to precisely one of the sets $w_{x+s}$ and $w_{x-s}$, say $w_{x+s}$, then $\left\{\eta \in w_{x+s}: \zeta \subset \eta\right\}=\left\{\eta \in w_{x-s}: \zeta \subset \eta\right\} \cup\left\{\eta^{\prime}\right\}$. In particular, $p\left(\left|\left\{\eta \in w_{x+s}: \zeta \subset \eta\right\}\right|\right) \neq p\left(\left|\left\{\eta \in w_{x-s}: \zeta \subset \eta\right\}\right|\right)$ implying that $g(x-s)\left(n_{x}\right) \neq$ $g(x+s)\left(n_{x}\right)$.

So, we can assume that $\eta^{\prime} \in w_{x-s} \cap w_{x+s}$. Then $\left\{\eta \in w_{x-s}: \zeta \subset \eta\right\}=$ $\left\{\eta \in w_{x+s}: \zeta \subset \eta\right\}$ and $\eta(x-s, \zeta)=\eta(x+s, \zeta)$. We will consider three cases.

CASE 1: $\eta^{\prime} \neq \eta(x-s, \zeta)=\eta(x+s, \zeta)$. Then $q_{x-s, \eta(x-s, \zeta)}+q_{x+s, \eta(x+s, \zeta)}=$ $0 ;$ so $q_{0}=0$ separates $q_{x-s, \eta(x-s, \zeta)}$ and $q_{x+s, \eta(x+s, \zeta)}$. Thus $k_{n_{x}}\left(q_{x-s, \eta(x-s, \zeta)}\right) \neq$ $k_{n_{x}}\left(q_{x+s, \eta(x+s, \zeta)}\right)$ and $g(x-s)\left(n_{x}\right) \neq g(x+s)\left(n_{x}\right)$.

CASE 2: $\eta^{\prime}=\eta(x-s, \zeta)=\eta(x+s, \zeta)$ and $q_{x-s, \eta(x-s, \zeta)} \neq q_{x+s, \eta(x+s, \zeta)}$. Then $q_{x-s, \eta(x-s, \zeta)}+q_{x+s, \eta(x+s, \zeta)}=2 q_{x, \eta^{\prime}}$ and, by the definition of $n_{x}, q_{x, \eta^{\prime}} \in$ $\left\{q_{j}: j<n_{x}\right\}$. Since $q_{x, \eta^{\prime}}$ separates $q_{x-s, \eta(x-s, \zeta)}$ and $q_{x+s, \eta(x+s, \zeta)}$, we conclude that $k_{n_{x}}\left(q_{x-s, \eta(x-s, \zeta)}\right) \neq k_{n_{x}}\left(q_{x+s, \eta(x+s, \zeta)}\right)$ and $g(x-s)\left(n_{x}\right) \neq g(x+s)\left(n_{x}\right)$.

CASE 3: $\eta^{\prime}=\eta(x-s, \zeta)=\eta(x+s, \zeta)$ and $q_{x-s, \eta(x-s, \zeta)}=q_{x+s, \eta(x+s, \zeta)}$. Then $Z=\left\{\eta \in w_{x-s}: \zeta \subset \eta\right\} \backslash\{\eta(x-s, \zeta)\}=\left\{\eta \in w_{x+s}: \zeta \subset \eta\right\} \backslash\{\eta(x+s, \zeta)\}$ is non-empty, since it contains $\hat{\eta}$, and so $\xi(x-s, \zeta)=\xi(x+s, \zeta) \notin w_{x}$. Therefore, as in Case 1, $q_{x-s, \xi(x-s, \zeta)}+q_{x+s, \xi(x+s, \zeta)}=0$; so $q_{0}=0$ separates $q_{x-s, \xi(x-s, \zeta)}$ and $q_{x+s, \xi(x+s, \zeta)}$. Thus $k_{n_{x}}\left(q_{x-s, \xi(x-s, \zeta)}\right) \neq k_{n_{x}}\left(q_{x+s, \xi(x+s, \zeta)}\right)$ and $g(x-s)\left(n_{x}\right) \neq g(x+s)\left(n_{x}\right)$.

This finishes the proof of (1).

Next, for every $x \in \mathbb{R}$ put $\delta_{x}=2^{-n_{x}}$. To finish the proof of the theorem it is enough to show that every $\hat{S}_{x}$ defined for such a choice of $\delta_{x}$ is a subset of a finite set

$$
Z_{x}=\left\{s \in \mathbb{R}: w_{x+s} \subset w_{x} \& n_{x+s}<n_{x}\right\}=\left\{\sum_{\eta \in w_{x}} p_{\eta} y_{\eta}: p_{\eta} \in\left\{q_{j}: j<n_{x}\right\}\right\} .
$$

Indeed, take an $s \in \hat{S}_{x}$. Then, by (1) and the definition of the distance function $d$, we have $\max \left\{n_{x-s}, n_{x+s}\right\}<n_{x}$. Notice also that if $n_{x-s} \neq n_{x+s}$, say $n_{x-s}<n_{x+s}$, then $g(x-s)\left(n_{x+s}\right)=\emptyset \neq g(x+s)\left(n_{x+s}\right)$ implying that $d(g(x+s), g(x-s)) \geq 2^{-n_{x+s}}>2^{-n_{x}}=\delta_{x}$, which contradicts $s \in \hat{S}_{x}$. So, we have $n_{x-s}=n_{x+s}$. To prove that $s \in Z_{x}$ it is enough to show that 
$w_{x+s} \subset w_{x}$. But if it is not the case, then there exists an $\eta \in w_{x+s} \backslash w_{x}$. Moreover, $q_{x+s, \eta}=-q_{x-s, \eta} \neq 0$ and $\eta=\eta(x+s, \zeta)=\eta(x-s, \zeta)$, where $\zeta=\eta\left\lceil n_{x+s}\right.$. In particular, $q_{0}=0$ separates $q_{x+s, \eta(x+s, \zeta)}$ and $q_{x-s, \eta(x-s, \zeta)}$. Therefore $k_{n_{x+s}}\left(q_{x-s, \eta(x-s, \zeta)}\right) \neq k_{n_{x+s}}\left(q_{x+s, \eta(x+s, \zeta)}\right)$ and $g(x-s)\left(n_{x+s}\right) \neq$ $g(x+s)\left(n_{x+s}\right)$. So $d(g(x+s), g(x-s)) \geq 2^{-n_{x+s}}>2^{-n_{x}}=\delta_{x}$ again contradicting $s \in \hat{S}_{x}$. Thus, $w_{x+s} \subset w_{x}$ and $s \in Z_{x}$.

\section{References}

[1] K. Ciesielski, On range of uniformly antisymmetric functions, Real Analysis Exch. 19(2) (1993-94), 616-619.

[2] K. Ciesielski, Uniformly antisymmetric functions and $K_{5}$, Real Analysis Exch. 21(2) (1995-96), 147-153. (Preprint* ${ }^{\star}$ available. ${ }^{1}$ )

[3] K. Ciesielski, Sum and difference free partitions of vector spaces, Colloq. Math. 71 (1996), 263-271. (Preprint* available.)

[4] K. Ciesielski, Set Theory for the Working Mathematician, London Math. Soc. Student Texts 39, Cambridge Univ. Press 1997.

[5] K. Ciesielski, Set theoretic real analysis, J. Appl. Anal. 3(2) (1997), 143190. (Preprint ${ }^{\star}$ available.)

[6] K. Ciesielski, L. Larson, Uniformly antisymmetric functions, Real Analysis Exch. 19(1) (1993-94), 226-235.

[7] P. Kostyrko, There is no strongly locally antisymmetric set, Real Analysis Exch. 17 (1991-92), 423-425.

[8] P. Komjáth, S. Shelah, On uniformly antisymmetric functions, Real Analysis Exch. 19(1) (1993-94), 218-225.

[9] Brian Thomson, Symmetric Properties of Real Functions, Marcel Dekker, 1994.

\footnotetext{
${ }^{1}$ Preprints marked by * are available in electronic form from Set Theoretic Analysis Web Page: http://www.math.wvu.edu/homepages/kcies/STA/STA.html
} 